\title{
OTC-27294-MS
}

\section{Technical and Economic Readiness Review of CFD-Based Numerical Wave Basin for Offshore Floater Design}

Jang Whan Kim, Hyunchul Jang, Aldric Baquet, Jim O'Sullivan, Technip; Sungwook Lee, Booki Kim, Samsung Heavy Industries Co., Ltd;

Alex Read, CD-adapco; Hrvoje Jasak, Wikki;

Copyright 2016, Offshore Technology Conference

This paper was prepared for presentation at the Offshore Technology Conference held in Houston, Texas, USA, 2-5 May 2016.

This paper was selected for presentation by an OTC program committee following review of information contained in an abstract submitted by the author(s). Contents of the paper have not been reviewed by the Offshore Technology Conference and are subject to correction by the author(s). The material does not necessarily reflect any position of the Offshore Technology Conference, its officers, or members. Electronic reproduction, distribution, or storage of any part of this paper without the written consent of the Offshore Technology Conference is prohibited. Permission to reproduce in print is restricted to an abstract of not more than 300 words; illustrations may not be copied. The abstract must contain conspicuous acknowledgment of OTC copyright.

\section{Abstract}

As Computational Fluid Dynamics (CFD) and High Performance Computing (HPC) technologies matured in many other industries, the offshore industry has begun to recognize CFD-based Numerical Wave Basin (NWB) as a design tool to evaluate offshore floater design more efficiently and with less uncertainty than the conventional ways relying on empirical methods. The recent NWB technology development has focused on the customization of CFD software for offshore design practices and validation of the developed analysis tools/procedures against physical model tests. Development has now extended to simulation of fully coupled hull-mooring-riser systems.

Technology readiness of the NWB for field application is demonstrated for two benchmark problems:

1. Vortex-induced motion of a multi-column floater

2. Global performance of a multi-column floater in extreme wave environment

The results indicates that the CFD-based numerical wave basin, although still computationally expensive, is technically ready to be a complementary tool to physical wave basin for offshore platform global performance design.

\section{Introduction}

CFD is now a ubiquitous part of the engineering design process. In their desire to reduce cost and project time, or time-to-market, industries ranging from automotive to rotating machinery have steadily increased their reliance on CFD simulations. For example, in a modern car, everything from the aerodynamic and engine performance to rain management is designed using CFD. For example, in an effort that is not atypical in the industry, Jaguar Land Rover (JLR) is working to fulfil their 2020 vision: complete a vehicle design sign-off based only on virtual design. This is no small undertaking, since today's vehicles are highly complex with roughly 9,000 customer requirements and 3,000 assessments needed for sign-off, and JLR are significantly increasing the number of "product actions". The business drivers and challenges, as laid-out by JLR's Head of Simulation Andy Richardson, could easily come from an O\&G: develop new technologies while managing massively increased system complexity; identify failure modes and establish countermeasures to achieve right first time design; reduce in-service failures; simulate the full range of use cases; optimized product, design efficiency and reduced production costs. 
CFD is already an integral part of the O\&G engineering toolkit. Usage can be placed into four categories: (1) equipment design from drill bits through Fluidized Catalytic Crackers or FCCs in a refinery; (2) understanding operational questions such as how best to produce a given reservoir, or how to manage well shut-ins to prevent solids forming and plugging pipelines or equipment; (3) safety including scenario analyses; and (4) troubleshooting equipment in the field. In this time of low oil prices, cost reduction is front and center for all. Simulation is playing a key role to reduce spending on physical testing, deliver projects on time and budget (manage CAPEX), and reduce operational expenses (OPEX) by delivering designs that are right the first time

In shipbuilding industry, CFD is extending its role to new hull-form design where traditional way encounters technical issues such as

1. Prediction of the resistance and self-propulsion performance at the initial design stage.

2. Prediction of the manoeuvrability and added resistance with navigation profile

3. Design of ship propellers

4. Development of Energy Saving Device (ESD).

Scaled model test is a traditional approach to evaluate hull performance at the initial design stage, and requires additional effort to prepare the test set-up and to analyze the test results. A regression model drawn from model test results is used to update designs due to inevitable design basis changes. However, the regression model is not reliable when the design parameters exceed the range of the test results. To overcome the limitation of the regression model approach, researchers have used CFD simulations for resistance, manoeuvrability, added resistance, and the development of the ESD, especially at the initial design stage. Toxopeus (2011) studied an application of CFD simulation to predict ship manoeuvrability and showed the possibility of the CFD simulations to replace model tests. Cura et al. (2006) and Sakamoto et al. (2012) performed CFD simulations of planar motion mechanism (PMM) test. Lee et al. (2015) studied the manoeuvrability of unconventional ships such as wind turbine installation vessel (WTIV). Kim et al. (2015) studied added resistance and the relationship between speed reduction and sea-states of a Container Ship in waves with RANS turbulence model. Choi et al. (2014) performed the optimization of the bulbous bow of a 9,000 TEU container ship with RANS, and improved the added resistance by 9\%. Lee et al. (2015) developed an ESD to improve the energy efficiency and satisfy the Energy Efficiency Design Index (EEDI) which took effect in 2013, and validated the simulation result with model test and full-scale sea trial.

Offshore industry has been continually improving analytic capabilities of global performance analysis, to provide accurate prediction of global motion and loading on floating structures including topsides, hull and mooring/riser in design environments. The most commonly used analytic tools are based on a semi-empirical approach combining linear and secondorder radiation-diffraction theory and Morison equation, calibrated by scaled physical model test. However, because of the limitations in the theoretical models, the offshore floater design still heavily relies on the scaled physical model test for the design parameters such as vortex-induced motion (VIM), ringing, air gap, green water and wave impact.

CFD had long been regarded as alternative approach to replace or reduce reliance on the physical model test in the design process. Unlike the conventional global performance analysis tools, the accuracy of the CFD solution solely depends on the mesh size and time step for the numerical solution with a proper choice of turbulence model and numerical methods, which has been proven from the extensive Verification and Validation (V\&V) work performed in other industries. However, the application of CFD for offshore floater design has not been successful yet until very recent. During floater design process, offshore engineers deal with the following unique challenges compared with other industries:

1. Highly-separated flow at high Reynolds number (order of $10^{7}$ ) around floater hull

2. Large scale difference between hull and mooring/riser

3. Open fluid domain to model ocean environment

4. Non-Gaussian stochastic environment and floater response

The high Reynolds number requires higher resolution for the flow near the structure surface and wake. Open fluid domain requires large fluid volume to be modeled. Non-Gaussian stochastic environment requires long simulation time, e.g. multiple realizations of typical three-hour storm duration, to provide reliable statistics of the floater motion and load responses. Massive parallel computing is inevitable to meet the computational requirements. Even with peta-scale (10 ${ }^{15}$ FLOPS) modern HighPerformance Computing (HPC) power, resolving spatial and temporal scales of physics involved in the whole Ocean-hullmooring/riser system by CFD alone is not practical yet.

A hybrid method where ocean waves are modeled by potential-based numerical wave tank and mooring and riser by slender Morison members is proposed as a practical approach. The coupling between potential-based numerical wave and CFD 
solution is achieved by matching the two solutions along the outer boundary and volume surrounding the CFD domain (Kim et al., 2012; Jacobson et al., 2012; Vukcevic and Jasak, 2015), or by decomposing numerical solution by incident wave solution from potential theory and scattered and radiating wave solution from CFD (Luquet et al., 2007). For mooring and riser, a finiteelement method based on rod dynamics (Webster et al., 2012) has been widely used in the conventional time-domain global performance analysis. Hydrodynamic force on the rods are modeled by Morrison equation where sectional drag and inertial coefficients are obtained from either model test or CFD simulation. The coupling of potential-based numerical wave and CFD solution near the offshore structure has been validated in many recent studies (e.g., Bockmann et al., 2014; Wu et al., 2014). The coupling of mooring/riser model and CFD solution have recently been validated in global performance of Semisubmersibles and TLPs (Wu et al., 2016; Teng et al., 2016).

The application of the CFD technology for offshore floater design is now maturing by implementing offshore customizations such as far-field wave modeling to confine CFD domain and finite-element modeling of mooring and risers. This paper addresses the technology and economic readiness of the combined system, which is referred to as CFD-based Numerical Wave Basin (NWB), by demonstrating validation against physical model test results and cost analysis of the two benchmark problems.

Firstly, the validation analysis of the following two problems are performed:

1. VIM model test of a multi-column floater in a towing tank

2. In-place model test (3-hr irregular wave test) of a multi-column floater in a wave basin with truncated mooring/riser model

Motion response, air gap and mooring/riser tension are compared against the model test data to demonstrate validation of the CFD-based NWB both in component and system level. For each benchmark problem, an additional simulation is performed where the truncated mooring/riser model is replaced by full-scale mooring/riser model to verify the validity of the model test set up with the truncated model.

Based on the full-scale analysis results and cost of the performed simulations, the economics of the CFD-based NWB technology for the offshore floater design are discussed based on the following cost-benefit analysis:

1. Overall cost compared with physical model test

2. Potential improvements in floater design

3. Impact on floater design schedule

The status review of the CFD-based Numerical Wave Basin technology based on valiation and cost-benefit analysis presented in this paper will provide a guidance on whether this new technology is ready to replace the role of physical model test in the offshore floater design process.

\section{Description of CFD-Based Numerical Wave Basin}

\section{CFD Solver}

A commercial CFD software, Star-CCM+ and an open source CFD code, OpenFOAM (Naval-Hydro Pack) are used in our numerical wave basin development. The following is a brief description of the CFD codes.

\section{$\underline{\text { Star-CCM+ }}$}

A commercial CFD software, Star-CCM+, provides a variety of turbulence models including families of Reynolds Averaged Navier-Stokes (RANS), Large-Eddy Simulation (LES), and Detached-Eddy Simulation (DES). In our recommended practice, DES is preferred for Vortex-Induced Motion (VIM) of floaters, and both of DES and RANS are relavant for global performance of floaters, but LES is still too expensive even in the model scale.

Variety of options of dynamic meshing strategy are available for hull motions, such as moving mesh, sliding mesh, mesh morphing, and overset grid techniques. For the single floating hull motion, a simple moving mesh technique, which is called Dynamic Fluid Body Interaction (DFBI) in Star-CCM+, is preferred due to its computational efficiency. The whole mesh system is translated and rotated following the rigid body motion of the floater. However, the accuracy of the solver at the domain boundaries degrades if rotational motion is significantly large. In the present global performance simulations, an algebraic mesh morphing is applied. 
Both air and water are modeled as incompressible fluids with the free surface. The free surface is captured using Volume-of-Fluid (VOF) technique with high resolution interface capturing (HRIC) scheme.

\section{OpenFOAM Naval Hydro Pack}

The Naval Hydro Pack is an OpenFOAM-based tool for off-shore and naval hydrodynamics simulations developed by Wikki. The tool uses an optimised formulation of a sharp free surface capturing solver with specific extensions for naval hydrodynamics use. The pressure-velocity equation set is solved in the mixture formulation (solving for velocity as a primitive variable rather than momentum) and the $\mathrm{p}-\mathrm{U}$ coupling includes the implicit implementation of the interface jump conditions (Vukcevic and Jasak, 2015). Free surface capturing is performed with either the implicit compression Volume-of-Fluid (VOF) type formulation or via the level set method in the correction form. Turbulence is modelled through the interface to OpenFOAM's turbulence library, including RANS and LES models; in this study, the k-omega SST model by Menter and Detached Eddy Simulation has been used.

Far field (wave) boundary conditions are introduced using the implicit relaxation zone approach in the free surface and momentum equations (Vukcevic and Jasak, 2015), where the free surface Navier-Stokes equation set is implicitly blended with the far field "wave theory", prescribing the desired far field behaviour of the wave field. The wave theory library includes support for regular and irregular waves, mean current, seabed boundary layers and is incorporated into the CFD solution via sub-cell resolution blending. Irregular waves are modelled either according to specified sea spectra (Bretschneider, PiersonMoskowitz, JONSWAP), as user-defined wave packets of varying wave height and wave number or via coupling to the nonlinear potential Higher Order Spectra solver implemented in the Naval Hydro pack. There also exists a possibility of coupling to as wave libraries, such as the one used in this study.

Floating body motion is resolved using the integrated 6-Degrees-of-Freedom (6-DOF) Ordinary Differential Equation (ODE) solver, which includes a combination of free and "prescribed motion" components and includes forces on the hull. The ODE is solved in a strongly coupled manner using a 5th order Kaps-Rentrop Runge-Kutta solver with automatic time-stepping. The interface to the mooring model is included, which prescribes mooring forces and moments based on the position and motion of a floating body.

Dynamic mesh motion in the Naval Hydro Pack is handled in a number of ways, including algebraic and elliptic mesh deformation as well as prescribed solid motion of the body. The Naval Hydro Pack includes support for massive parallel execution with efficiency of the same order as commercial CFD tools.

\section{Coupling CFD with Mooring/Riser and Wave Libraries}

Since the application of CFD for offshore floater is relatively new compared to the other industrial applications, some functionalities for offshore application, e.g. modeling of input wave and mooring/riser model, are not fully developed yet to the level of standards in other industries. The name of the CFD-based numerical wave basin tool implemented by Technip is called MrNWB (Mooring/Riser Numerical Wave Basin). The MrNWB consolidates three components such as CFD solver, mooring/riser model, and wave models. The mooring/riser and wave models use a shared library format, which can be dynamically linked to the CFD solver. Three wave models have been used so far: regular wave based on inverse formulation or irrotational Green-Naghdi equation, linear irregular wave, and nonlinear irregular wave. The MrNWB was developed by Technip based on a commercial CFD software, Star-CCM+. The same shared libraries were also applied to an open-source CFD code, OpenFOAM (Naval-Hydro pack) by Wikki in a joint development project with Technip, Samsung Heavy Industries Co., Ltd and Wikki.

The potential-based wave models are coupled with the commercial CFD solver, Star-CCM+, using the Euler-Overlay Method (EOM) (Kim et al, 2006; 2012). The wave solutions from the potential based wave library are applied to the outer domain, and the fully non-linear wave solutions are obtained from CFD solver in a smaller domain containing the floating structure. The overlay zone located between the outer Euler zone and the inner CFD zone gradually blends the CFD solutions and the Euler solutions applying source terms to VOF and momentum equations. Figure 1 explains the idea of the EulerOverlay method. The OpenFOAM used the relaxation method developed by Vukcevic et at. (2015), which is a similar idea to the EOM. The introduction of the EOM reduces the computational cost drastically by confining CFD domain near the floater. The smaller CFD domain also helps to minimize wave attenuation due to numerical damping.

Figure 2 illustrates how the MrNWB communicates with CFD solver, wave library, and mooring/riser library. The CFD solver obtains the wave kinematics and source terms for EOM at each cell centroid from the wave library. It provides the 
body motion of the floater to the mooring/riser library and gets the resultant mooring forces/moments from the library. The mooring/riser library also gets the wave velocity and acceleration at the each finite element node from the wave library.

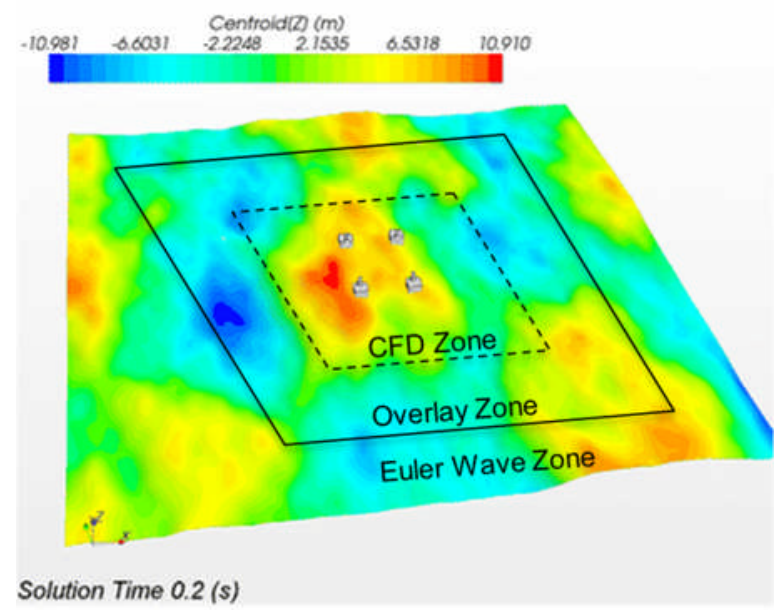

Figure 1: Illustration of Euler-Overlay Method (EOM)

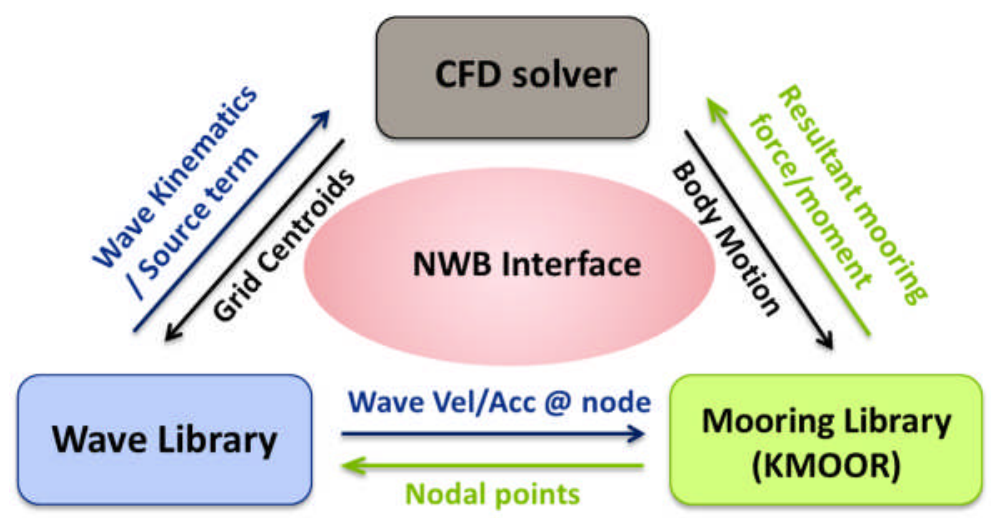

Figure 2: Structure of MrNWB (Mooring/Riser Numerical Wave Basin)

\section{Mooring and Riser Model}

The mooring line is modeled based on typical Finite Element modeling of dynamic catenary with lumped mass. It allows multiple segments of catenary models, which have not been provided by either commercial CFD softwares or opensource CFD codes. The hydrodynamic drag and inertia forces on the mooring/riser components are calculated based on empirical Morison formula. The resultant mooring forces and moments are obtained from the summation of forces and moments at the hang-off locations of the moorings and risers. The resultant mooring forces and moments are transferred to the 6DOF motion solver in the CFD software to update the body motion. The time step for the mooring/riser model is usually smaller than the CFD solver. Thus, the mooring model is calculated with the smaller sub-time steps at each CFD time step.

Viscoelastic model is used for the polyester mooring capability. The polyester mooring, which has been widely used with offshore floating platforms, has the time-dependent material properties such as creep, strain-stress hysteresis, and excitation period-dependent stiffness (Kim et al., 2010). In the model, the stretch of the polyester is given as the combination of quasi-static stiffness due to mean current, mean wind, and mean wave drift force and dynamic stiffness due to gust, wave, and VI M. Typical values of the dynamic stiffness and static stiffness are $30 \mathrm{MBL}$ and $13 \mathrm{MBL}$, respectively, where MBL is the minimum breaking load of the polyester rope. Because of the time delay in the response of the polyester rope, a relaxation time is introduced in the viscoelastic model - $120 \mathrm{~s}$ of the relaxation time is used in this study. 


\section{Potential-Based Numerical Wave Tank}

Technip's in-house potential-based numerical wave tank (PNWT) is used to generate 3-hour nonlinear irregular sea state. Wave libraries are built from the PNWT solution and are applied to the domain surrounding the CFD domain and the EOM method is applied to match the two different solutions smoothly.

PNWT is based on a finite-element approximation of the Hamilton's principle for the gravity wave in ideal fluid. Vertical profile of the velocity fields are assumed as 16-th order polynomial and piece-wise linear interpolation is used in horizontal direction. Time integration of the waves are made by Runge-Kutta 4-th order method. In addition, numerical wave breaking in PNWT is prevented by adaptive numerical damping mimicking the energy dissipation in physical wave breaking. Other wave models (such as High Order Spectral method) can also be used to build such wave libraries in the MrNWB.

One thousand linear wave components with random phases are generated and used as an input for the PNWT simulation. The wave components need to be calibrated to match the target wave spectrum (e.g. JONSWAP). Similarly to what is done in generating a particular sea state in a physical wave basin, a correction factor based on the ratio between the obtained spectrum from PNWT simulation and the target spectrum is calculated and applied to the input wave components. This calibration process usually requires a couple of iterations.

Forty 3-hour realizations with different seed numbers are simulated and analyzed efficiently using the PNWT. The best realizations can be chosen based on the wave spectrum and the wave statistics (Hs, Tp, skewness and kurtosis) and used for the CFD simulation.

\section{Validation of Numerical Wave Basin (Model of the Model)}

\section{Hull and Mooring Set-up}

Two deep draft semi-submersible hulls are used for Vortex-Induced Motion (VIM) and Global Performance (GP) simulations. Figure 3 shows the semi-submersibles such as HVS (Heave and VIM Suppressed) and conventional semisubmersibles. Both hulls are tested for the VIM simulations, and the HVS hull is tested for GP simulations. For VIM simulation, a planar linear mooring system is used with four linear springs. For the GP simulation, a truncated mooring system consisting of four taut mooring lines, one in each corner, is used. Both mooring systems in the CFD simulations are the same as the model tests. Detailed information of two hulls and mooring line properties for the VIM test and the GP test are given in Kyoung et al. (2015) and Kyoung et al. (2013), respectively. Relative wave elevations are measured also at the same location as in the model test. A sketch of the mooring set-up and the wave gauge locations for the HVS GP simulation is given in Figure 4.

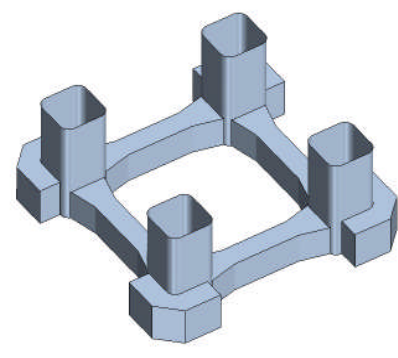

(a) HVS Semi.

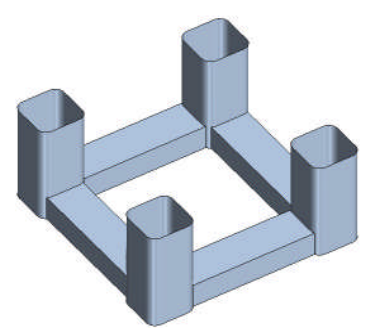

(b) Conventional Semi.

Figure 3: Semi-submersibles for VIM and GP Simulations 


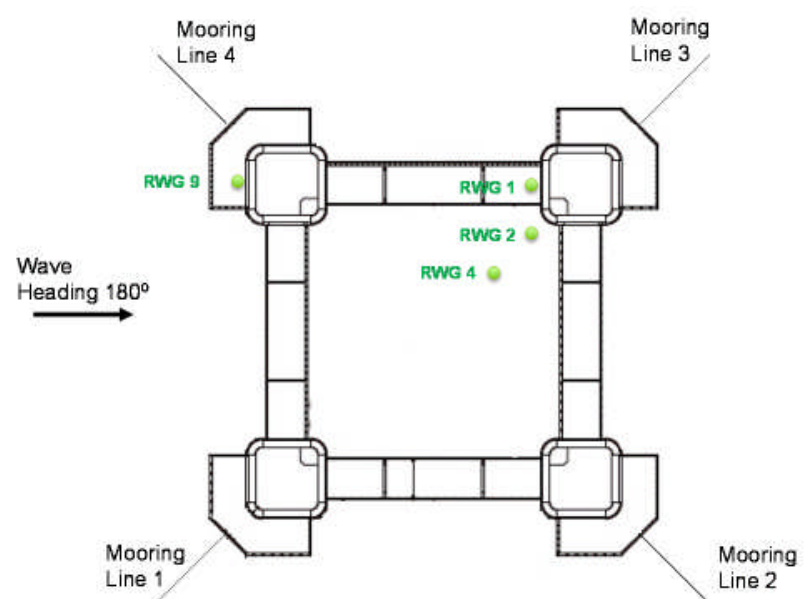

Figure 4: Mooring and Relative Wave Gauge Set-up for GP Simulation

\section{Input Wave Validation}

The sea state corresponding to a 1,000-year hurricane (i.e. a JONSWAP spectrum with $\mathrm{Hs}=17 \mathrm{~m}, \mathrm{Tp}=15.5 \mathrm{~s}$ and $\gamma=$ 2.6) was generated in PNWT and the solution was used in the domain surrounding the CFD domain in MrNWB. A wave only simulation was performed in CFD and the measured wave statistics and spectrum were analyzed at the target wave gauge to ensure that the simulated sea state matches the target spectrum. For reference, a CFD wave only simulation was also performed using linear wave components for the wave input. The wave spectra from three model test realizations and one CFD realizations are compared in Figure 5. One can see that the wave condition simulated in CFD matches quite well the model test and the target JONSWAP spectra. A better match is observed with the PNWT input wave compared to the linear input wave.

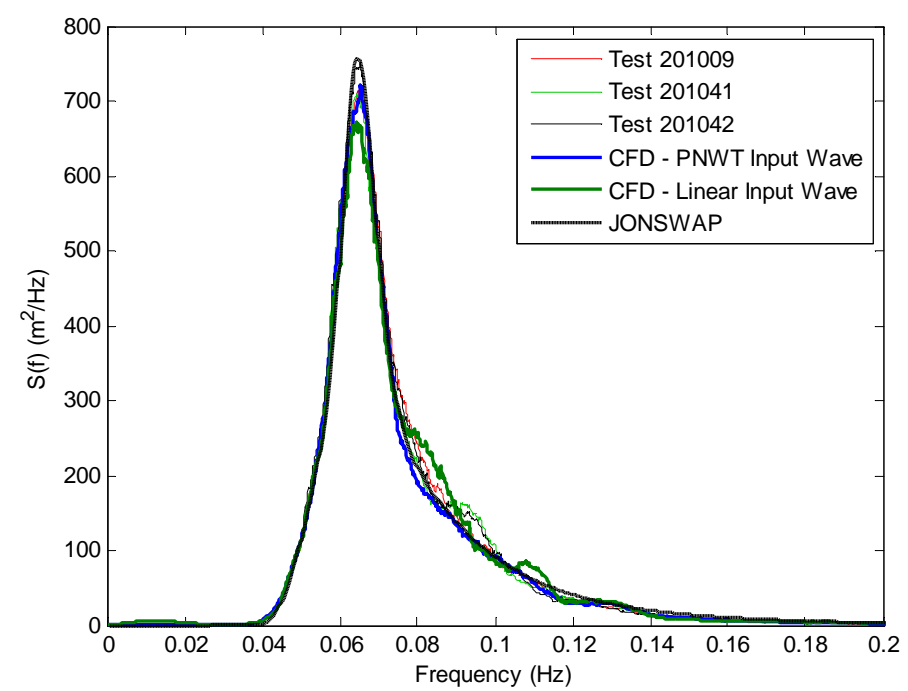

Figure 5: Wave Spectra from Model Test and CFD Simulation

\section{Vortex-Induced Motion (VIM)}

An offshore floater exposed to a steady current can suffer from the transverse motions due to the downstream formation of alternating vortices. This motion is referred as Vortex-Induced Motion (VIM). The VIM response can yield significant damage to the fatigue life of mooring and risers. Kyoung et al. (2015) validated the CFD simulations against the VIM model test for two semi-submersibles. Figure 6 shows the comparison of the nominal A/D*, which is defined by $\sqrt{ } 2 \times$ STD (Sway/D*) where $\mathrm{D}^{*}$ is the projected diameter of the column of the floater. The CFD shows excellent agreement to the model test for both semi-submersibles. The current CFD technology is able to predict the VIM motion of semisubmersible in good reliability. Furthermore, VIM performance for the HVS is significantly better than the conventional semisubmersible of the same draft. The mitigation mechanism of VIM response of the HVS was able to be successfully identified by the CFD simulation. 


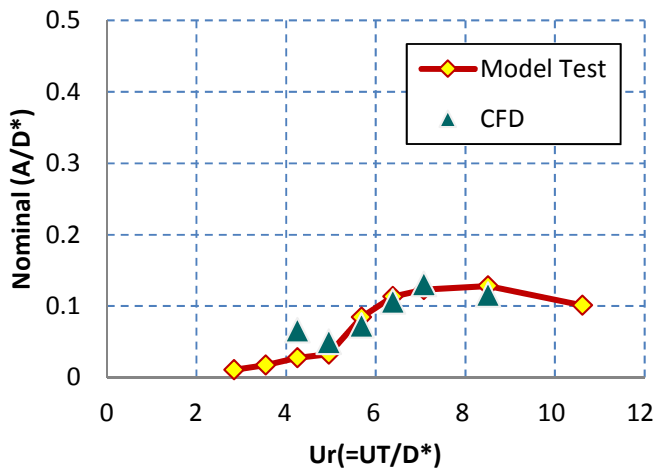

(a) HVS Semi: VIM response

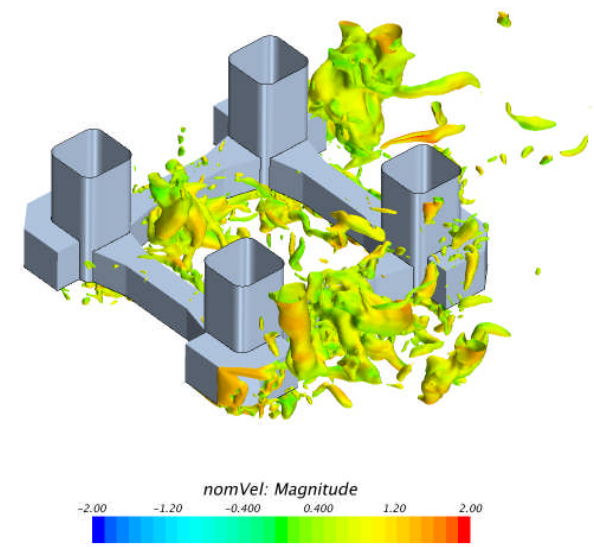

(c) HVS. Semi: Iso-pressure contour

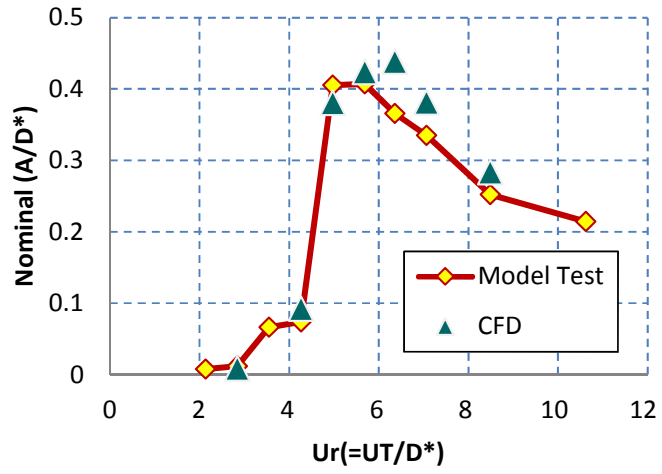

(b) Conv. Semi: VIM response

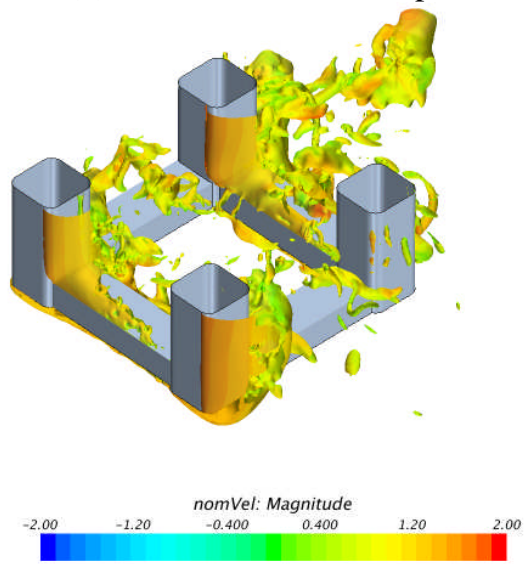

(d) Conv Semi: Iso-pressure contour

Figure 6: Validation of VIM Responses of Semisubmersibles at 45 deg Heading

\section{Global Performance in 1,000-yr Hurricane Wave Condition}

A global performance simulation was performed for the HVS semisubmersible in MrNWB using the 1,000-year hurricane wave presented in the previous input wave validation section. Similarly to the physical model test, system identification tests (i.e. equilibrium test, offset test and free decay tests) are performed to ensure that the model set-up is correct. For the equilibrium test, the free floating platform with mooring lines and risers is released in calm water until it reaches its equilibrium position. With this test, one can ensure that the model is well balanced at an even keel position. For the offset test, constant forces in the surge direction are applied to the model in calm water and the resulting offset is checked against theoretical values. The offset test curve for the studied semisubmersible is given in Figure 7(a). As one can see, the offset curve from CFD exactly matches the theoretical curve. This gives indications that the mooring restoring force is well modeled in CFD. For the free decay test, an initial displacement in one of the degrees of freedom (e.g. heave) is applied to the platform. The platform is then released at the beginning of the simulation and oscillates around its equilibrium position. With this test, one can verify the natural periods and the damping ratios of the system for the six degrees of freedom. In the heave decay test, the mean natural period from model test is $22.1 \mathrm{sec}$, and that from CFD is $22.0 \mathrm{sec}$. Damping ratios in the decay test from model test and CFD are compared in Figure 7(b). Both natural period and damping ratio from model test and CFD show good agreement. 


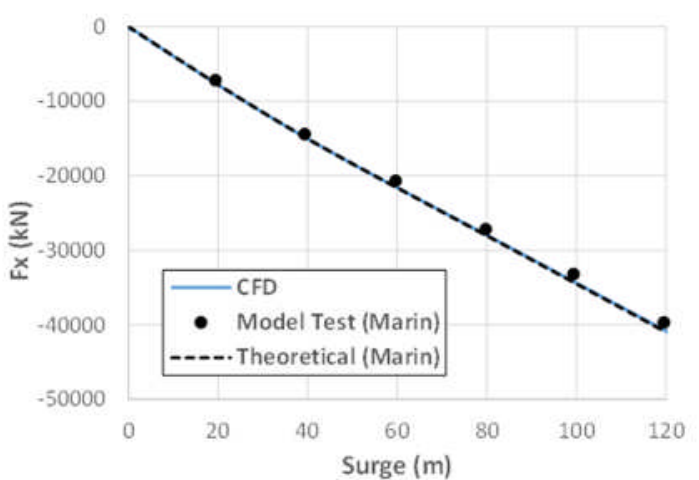

(a) Offset Test in Surge Direction

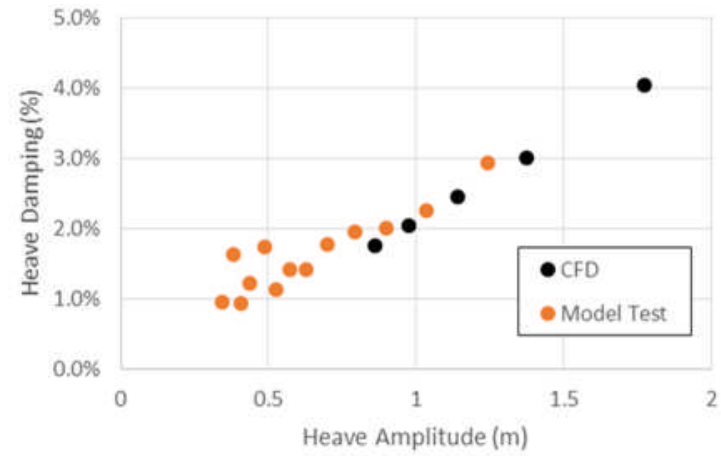

(b) Damping Ratio in Heave Decay Test

Figure 7: System Identification of HVS Semi GP Simulation

Results from a head sea simulation are presented in this section. Sensitivity tests on mesh size and time step have been performed to determine the optimal values. The mesh used in the simulation is represented in Figure 8. At the free surface the mesh size is $2 \mathrm{~m}$ horizontally and $1 \mathrm{~m}$ vertically. Near the body, the mesh size is $1 \mathrm{~m}$ both horizontally and vertically. In the studied case, head sea heading (i.e. $180 \mathrm{deg}$ heading) is analyzed. Since the mooring system is symmetric, the simulation is performed for a half model with symmetric boundary condition in $y=0$ plane as shown in Figure 4 . The number of cells for the half model is about 1.3 million. A time step of $0.04 \mathrm{~s}$ has been used in the simulation.

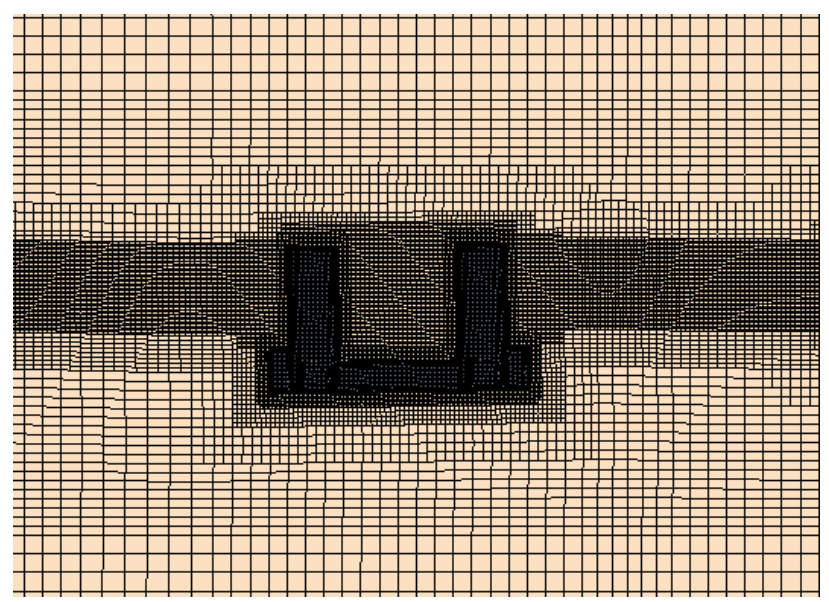

Figure 8: Computational Mesh for Head Sea Simulation

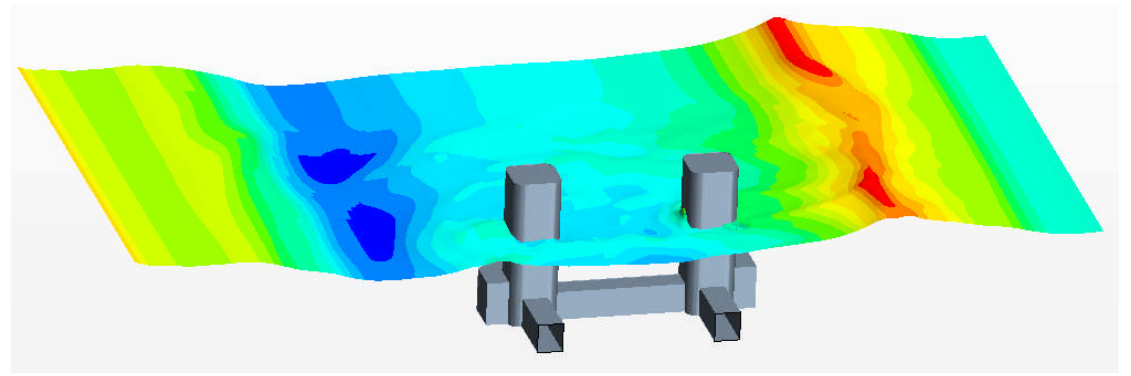

Figure 9: Snapshot of Half Model in Head Sea Simulation

A 3-hour simulation is performed in CFD for one wave realization with the sea state characteristics presented in the previous input wave validation section. Motions, mooring line tensions and relative wave elevation correlation factors obtained from the CFD and model test results are presented in Figure 10. The model results reported herein are the average of three 3hour tests (different wave realizations) while only one realization is used to report the CFD results. The extreme values (maximum and minimum) are the 3-hour extreme values from Weibull fitting with 50\% non-exceedance. In general, good 
correlation is observed between CFD and model test results. Especially, the ratios of standard deviations from CFD and model test are within $2 \%$ for all measured quantities.

Response spectra from both model test and CFD are compared in Figure 11 and Figure 12 for the tension in mooring line \#4 and the relative wave elevation measured at the wave gauge \#9, respectively. The mooring line tension spectrum from model test and CFD match quite well. Especially, as shown in Figure 11, the responses at surge natural period (212s), pitch natural period (36s), heave natural period (21s) and wave peak period (15.5s) are well captured in CFD. In addition, the good agreement of the relative wave elevation spectra from model test and CFD shown in Figure 12 also proves that MrNWB can be used for air gap prediction. More detailed information can be obtained in Wu et al. (2016).
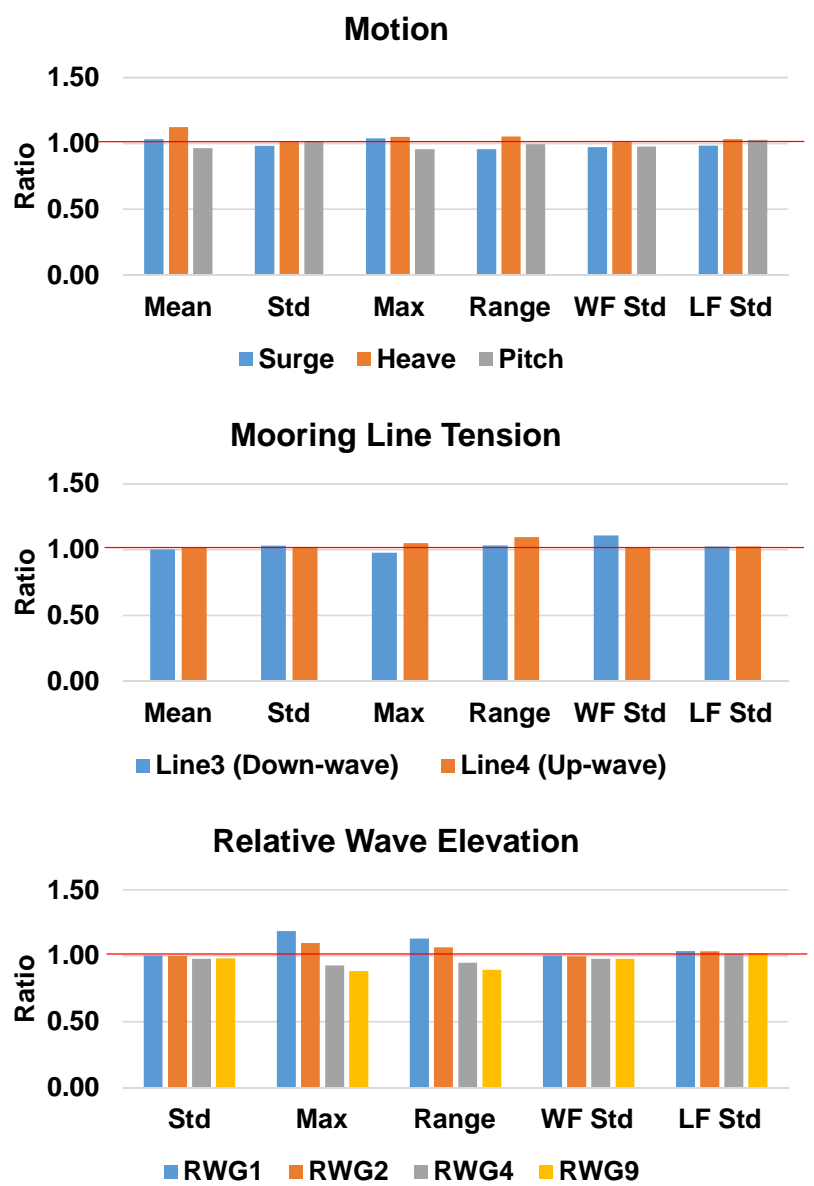

Notes:
(1) Ratio is defined as (CFD with Truncated Mooring Model value) / (Model Test value)
(2) Range is defined as Max - Min
(2) Cutf frequencies defined as: Wave Frequency $(\mathrm{WF})>(1 / 30 \mathrm{~s})$, Low Frequency $(\mathrm{LF})<(1 / 30 \mathrm{~s})$

Figure 10: Comparison of Motion, Mooring Line Tension, and Relative Wave Elevation from CFD and Model Test for HVS Global Performance in 1,000-year Hurricane Sea State 


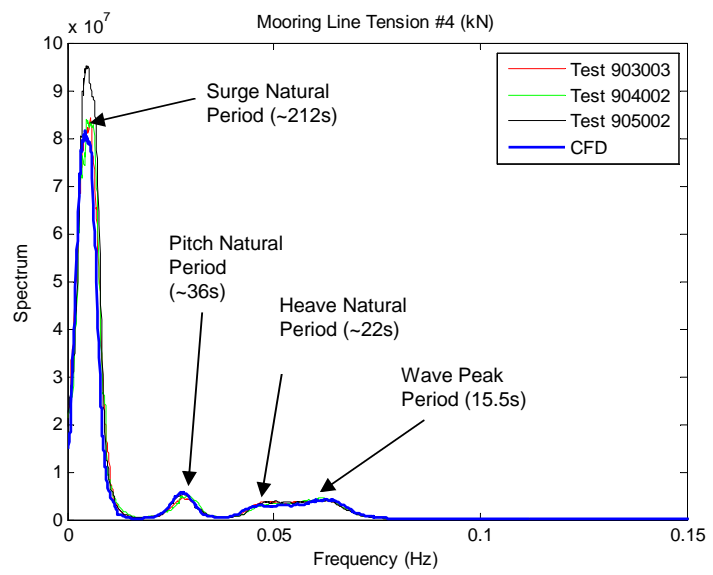

Figure 11: Mooring Line Tension Spectra in HVS GP Simulation

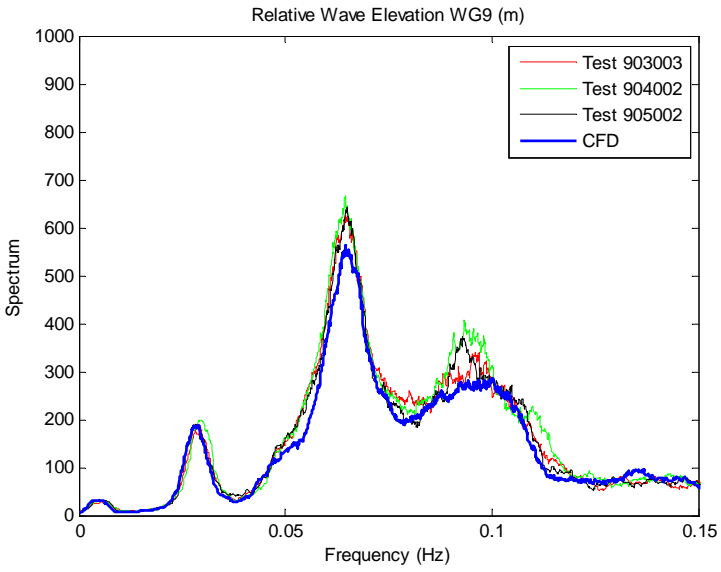

Figure 12: Relative Wave Gauge Spectra in HVS GP Simulation 


\section{Global Performance Using OpenFOAM}

A global performance simulation for the HVS semi-submersible was also performed by using OpenFOAM (NavalHydro Pack) with the MrNWB. The exact same computational mesh and time step are used for the OpenFOAM simulation as the Star-CCM+ simulation. The validation was performed in a regular wave case where the wave height is $14.22 \mathrm{~m}$ and the period is $13 \mathrm{~s}$. Figure 13 compares the motion time histories from model test and two CFD simulations done by Star-CCM+ and OpenFOAM. Both CFD simulations show fairly good agreement to the model test, and the OpenFOAM results looks quite similar to the Star-CCM+ results, but the OpenFOAM results slightly under-predicted the standard deviations of the motions.

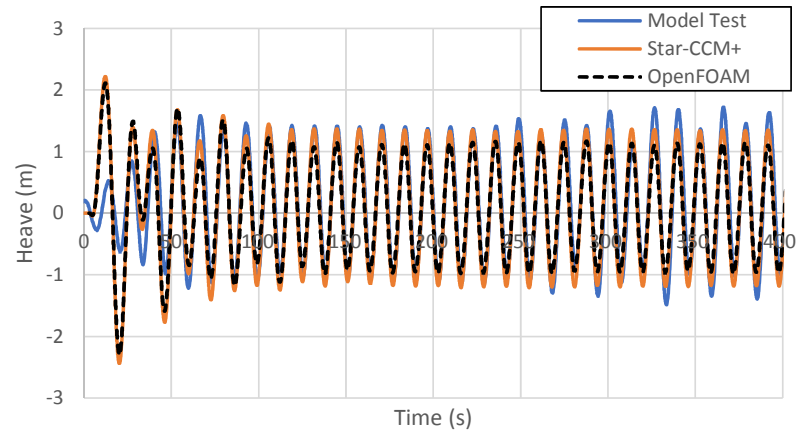

(a) Heave

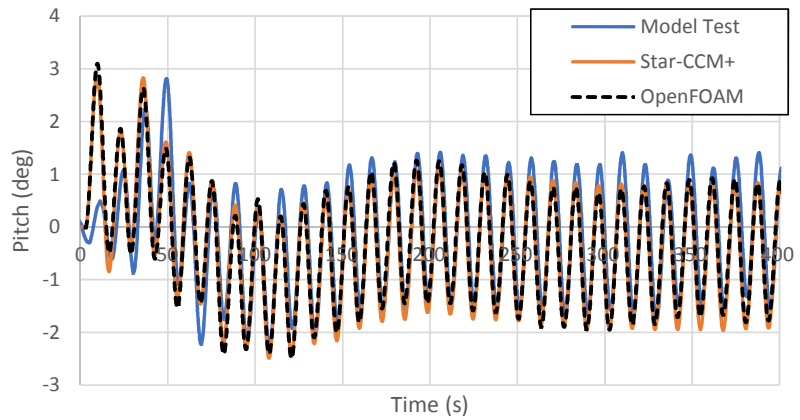

(b) Pitch

Figure 13: HVS GP Simulation in a Regular Wave.

\section{MrNWB Applications to Full-Scale Simulation}

The HVS semi-submersible is designed for $6,000 \mathrm{ft}(1,828.8 \mathrm{~m})$ water depth and moored by a total of 16 taut mooring lines, four at each column. The water depth in the wave basin is limited to $610 \mathrm{~m}$ at a scale of 1:61. Thus, a truncated and bundled mooring system was used in the model test to closely represent the stiffness characteristics of the full mooring system. The MrNWB enables us to perform both VIM and GP simulations with the full mooring system and viscoelastic polyester moorings. Figure 14 shows a schematic overview of the truncated mooring system (model test set-up) and the full depth mooring system. In the full mooring system, eight steel catenary risers are also added. In the figure, the black lines represent the mooring lines, and the red lines represent the steel catenary risers. 

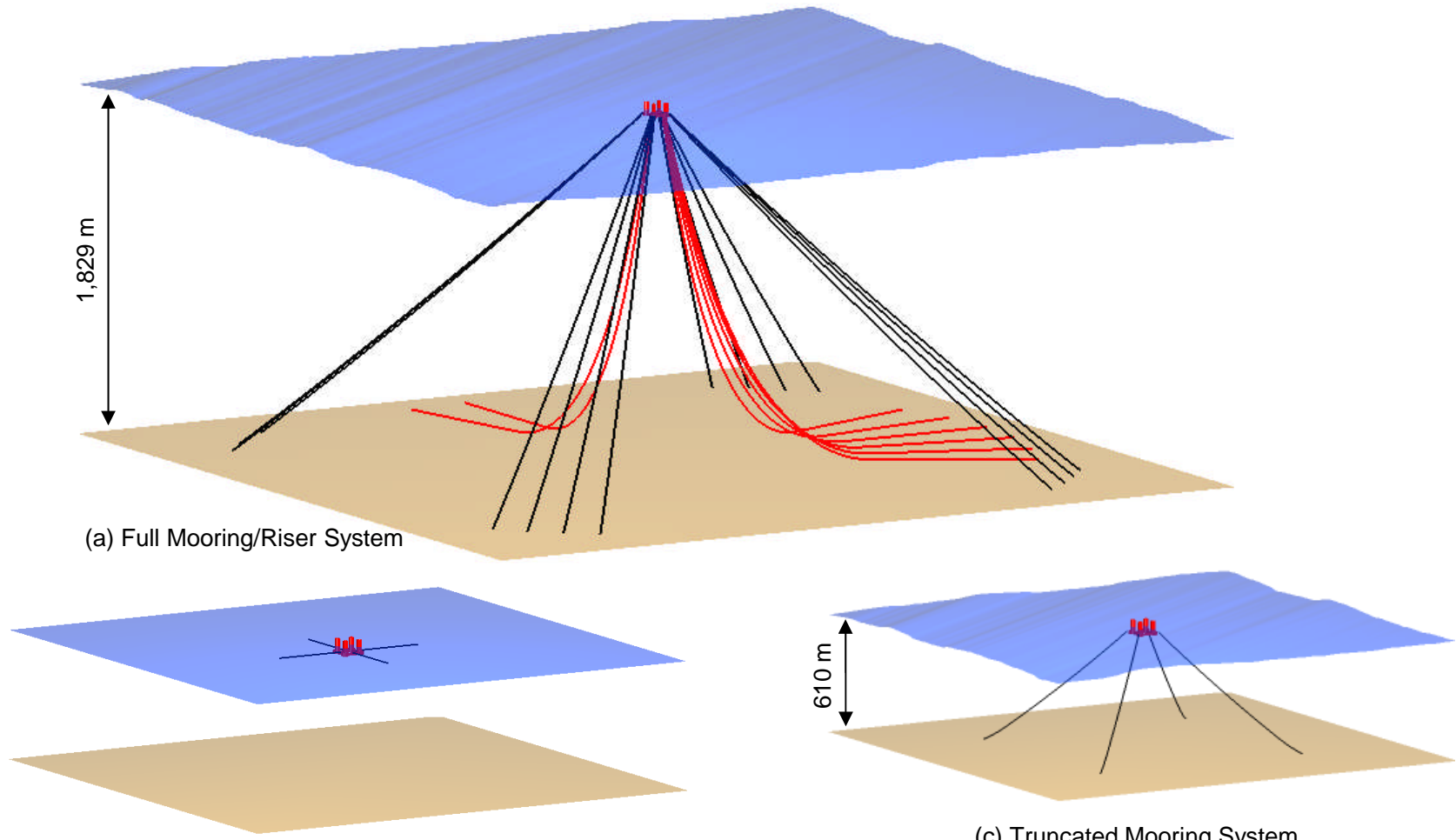

(b) Planar Mooring System used for VIM Model Test

(c) Truncated Mooring System used for In Place Model Test

Figure 14: Full and Truncated Mooring/ Riser Systems

\section{VIM with Full Mooring and Riser Model}

The full mooring system including viscoelastic polyester moorings and steel catenary risers is applied to the VIM simulation, and the results are compared to the planar linear spring mooring system used in the VIM model test. The VIM simulation is performed for the conventional semi-submersible case, which could be a good benchmark case to clearly show the difference from the mooring systems. Figure 15(a) compares the nominal A/D* plots from linear and full mooring systems. The VIM response from the full mooring system is significantly smaller than the linear mooring system, although it is still higher than the VIM response of HVS shown in Figure 6(a) without consideration of the full mooring system. The damping ratios from the full mooring/riser system are between 10 and $15 \%$ as shown in Figure 15(b).

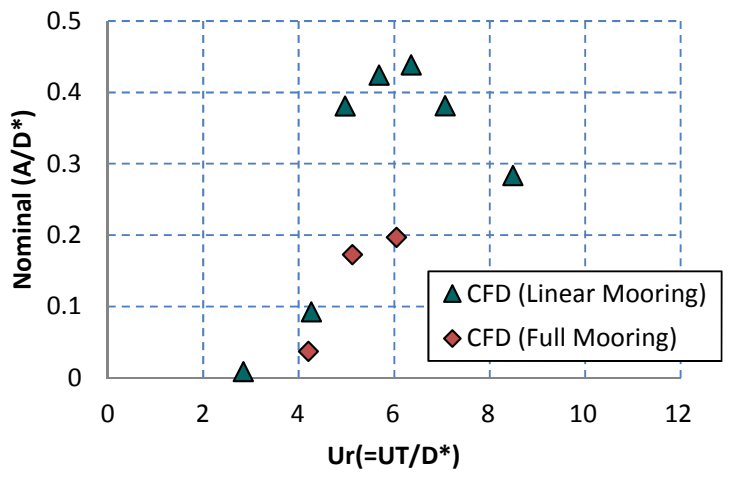

(a) Nominal A/D*

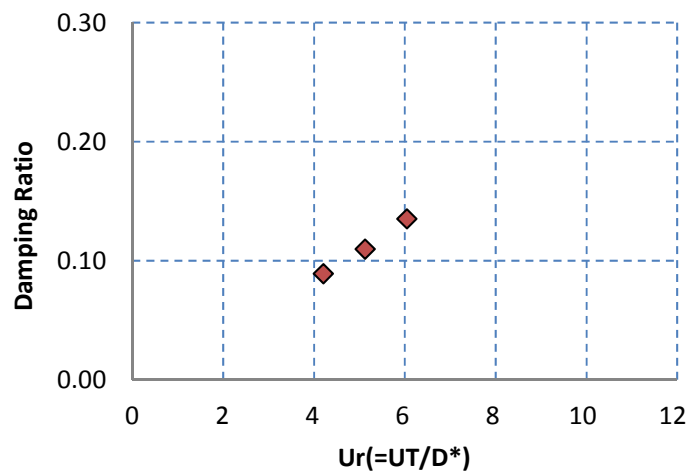

(b) Damping Ratio

Figure 15: VIM Reponses of Conventional Semi with Linear and Full Mooring Systems. 


\section{Global Performance with Full Mooring and Riser Model}

The full mooring system including viscoelastic polyester moorings and linear steel catenary risers is applied to the global performance analysis for the HVS semisubmersible in 1,000-year hurricane sea state. The motions and relative wave elevations from the head sea simulation are compared with the CFD results using the truncated mooring system in Figure 16. The heave response and the relative wave elevation results are quite consistent between the two models. The main effect of using the full mooring system and the full water depth is observed in the low frequency reponse. In particular, the pitch low frequency $(<1 / 30 \mathrm{~s})$ standard deviation for the full mooring model is $25 \%$ lower than the truncated mooring model. Also, the surge low frequency standard deviation for the full mooring model is $50 \%$ lower than the truncated mooring model. The pitch and surge spectrum comparison in Figure 17 clearly shows the lower response in the low frequency range for the full mooring system due to the damping.
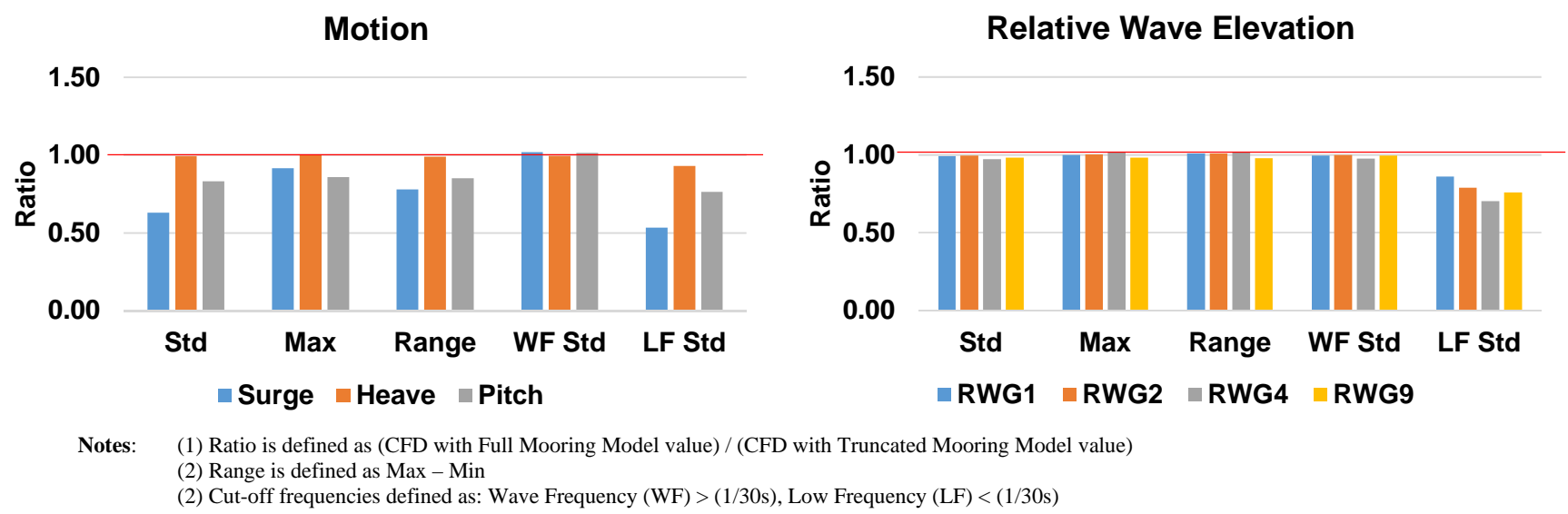

Figure 16: Comparison of Motion and Relative Wave Elevation from CFD Simulations with Full and Truncated Mooring Systems for HVS Global Performance in 1,000-year Hurricane Sea State
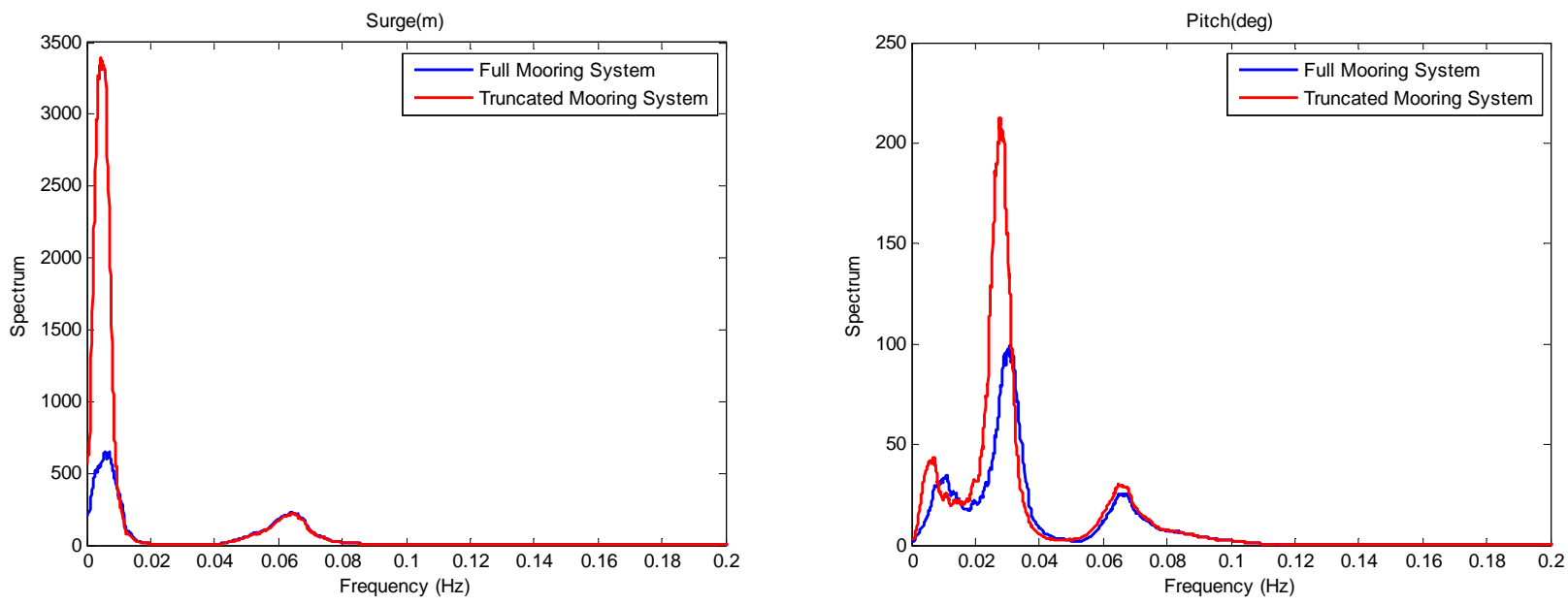

Figure 17: Surge and Pitch Response Comparisons for Full and Truncated Mooring System

\section{Cost \& Benefits of CFD-Based Numerical Basin}

Based on computing cost from the global performance simulation with full mooring/riser system, cost and time of a typical inplace global performance analysis can be estimated. The simulation cost is based on Star-CCM+ running on Stampede cluster in Texas Advanced Computing Center (TACC). Two days of computing time with 640 cores is assumed for each 3 hour simulations when estimating the simulation cost. The simulation cost based on OpenFOAM run has not been estimated yet due to lack of full 3-hour simulation yet but expected to be comparable to Star-CCM+ considering trade between lower software cost and higher man-hour cost. Other costs, including fabrication of model (or modeling \& verification in CFD) and man-hr cost is estimated based on Technip's previous experiences in model tests and CFD projects. 

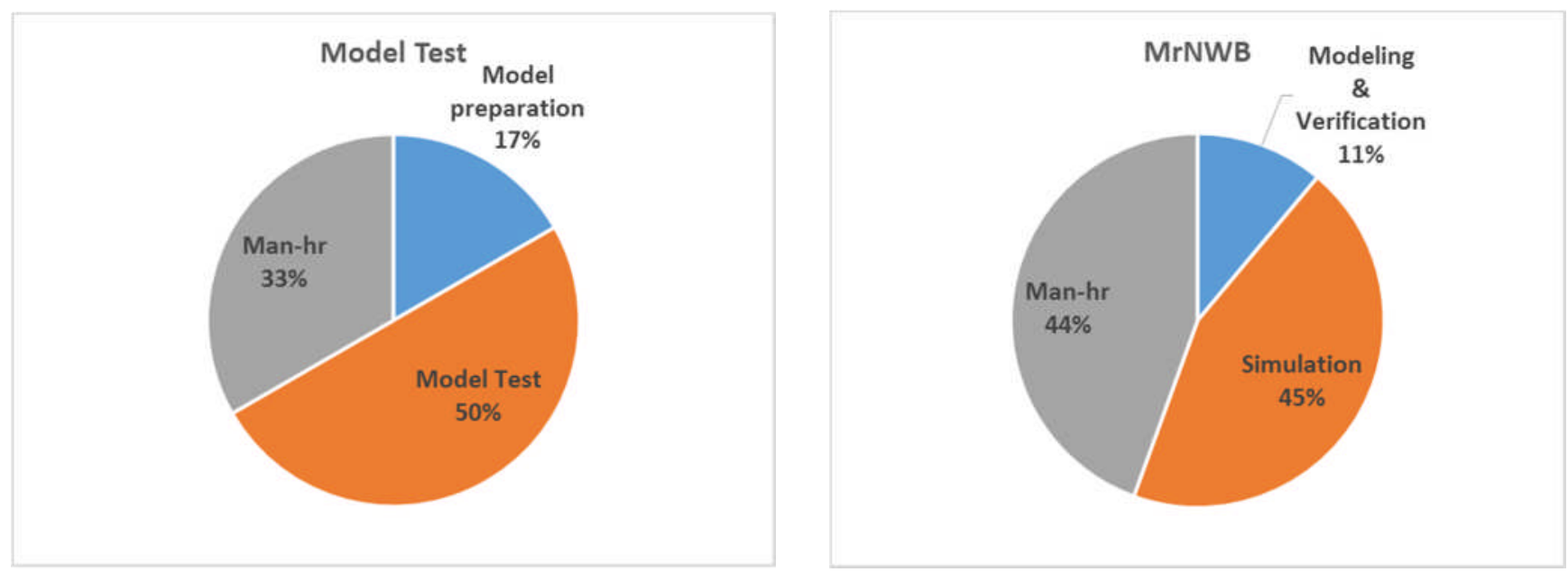

Figure 18: Cost Breakdown of Physical Model Test and NWB Simulation for Inplace Global Performance.

Typical inplace model test for Semi-submersible runs 80 test cases. The CFD project cycle for this scope of work and estimated time duration are

1. Proposal and tendering $(1-2$ months $)$

2. CFD model set up and verification (1 months)

3. Production runs (1 - 2 months)

4. Data processing and reporting (1 months)

After tendering of the project, CFD project takes $3-4$ months. It should be noted that the duration of production run depends on availability of computer resources. The time estimation here is based on 7\% utilization of total capacity of TACC Stampede supercomputer or 10 concurrent runs at a time, and 2 days of waiting time. Theoretically, one can make 156 concurrent runs with full capacity of the Stampede. The time duration of production run is more constrained by time for data review and checking rather than computing resources in this case.

The cost of CFD project is also estimated and found similar to that of physical model test. The cost breakdown shown in Figure 18 indicates that the unit cost of each simulation case is even lower than the unit cost of each model test, which means CFD can provide more test cases with less impact on total cost. Basin are

Based on the cost and time estimation exercise, the most expected benefits of using CFD-based Numerical Wave

1. Predictable and reliable time schedule and cost estimation

2. Quick turn-around time for changes in design and metocean data during the project

3. Lower cost and time for the second campaign of NWB simulation in case additional simulation is required

\section{Conclusions}

A CFD-based NWB coupling CFD solver with potential-based numerical wave tank and finite-element mooring/riser model are successfully applied to VIM and global performance in an extreme wave condition with full-scale mooring and riser layout. Cost and time comparison with physical model test shows benefits of CFD-based NWB in terms of more reliable and shorter project schedule, which will lead to savings in the offshore project cost.

It should be noted that the CFD-based NWB will continue to mature and provide more realistic field simulations. On going efforts toward the maturity are

1. Validations on combined environments of wave, wind and current in physical model test set up

2. Realization of combined environments of wave, wind and current in real sea 
3. Zooming analysis of wave impact events for slamming load estimation

\section{Acknowledgements}

The authors would like to thank Technip and Samsung Heavy Industries Co., Ltd for allowing and providing support to publish this paper, and Dr. Bonjun Koo, Dr. Ho-Joon Lim and Dr. Johyun Kyoung for their review and discussion on model test and simulation results. The authors also would like to thank continual and enthusiastic support from collaboration partners for the numerical wave basin development. Also appreciated are administrative and technical support from Texas Advanced Computing Center (TACC) for the Stampede use.

\section{References}

Bockmann, A., Kristiansen, T., Pakozdi, C., Jang, H. and Kim, J.W., "An Experimental and Computational Development of a Benchmark Solution for the Validation of Numerical Wave Tanks," OMAE2014-24710, San Francisco, USA, June 2014.

Choi, N.Y. Kim, H.T. Song, Y.Y. Choi, S.H. \& Kim, J.J., "Study on the Optimization of bulbous bow shape considering the Operating profile of ships," Proceeding of the society of Naval Architects of Korea, Busan, Republic of Korea, 22 23 May 2014. Cura, H.A., 2006, "Virtual PMM tests for manoeuvring prediction," In 26th Symposium on Naval Hydrodynamics, Rome, Italy, 2006, pp.31-49.

Jacobsen, N.G., Fuhrman, D.R. and Fredsøe, J., "A wave generation toolbox for the open-source CFD library: OpenFoam" International Journal for Numerical Methods in Fluids, vol 70, no. 9, pp. 1073-1088, 2012.

Kim, H.T. Hong, C.B. Lee, G.H. \& Kim, B.K., "Prediction of added resistance of a ship in waves using computational fludi dynamics," Proceeding of the society of Naval Architects of Korea, Jeju, Republic of Korea, 21 22 May 2015.

Kim, J. W., Kyoung, J. H., Ertekin, R. C. and Bai, K. J., “A Finite-Element Computation of Wave-Structure Interaction between Steep Stokes Waves and Vertical Cylinders", Journal of Waterway, Port, Coastal and Ocean Engineering, Vol. 132, No. 5, pp 337347, 2006.

Kim, J.W., Kyoung, J.H., Sablok, A., "Application of a Viscoelastic Model for Polyester Mooring," OMAE2010-20040, Shanghai, China, June 2010.

Kim, J.W., O'Sullivan, J. and Read A. "Ringing Analysis of a Vertical Cylinder by Euler Overlay Method," OMAE2012 84091, Rio de Janeiro, Brazil, June 2012.

Kyoung, J.H., Yang, C.K., O'Sullivan, J., and Miliante, T., "Validation of the HVS Semisubmersible Global Performance by Model Tests," OMAE2013-10402, Nantes, France, June 2013.

Kyoung, J.H., Kim, J.W., Jang, H., Lambrakos, K., O'Sullivan, J., "Investigation on the VIM mitigation of the HVS semisubmersible", OMAE2015-41188, St. John's, Canada.

Lee, H., Hong, C., Kim, H., Choi, S., Han, J., Kim, B. and Lee, J., "Development and Application of Energy Saving Devices to Improve Resistance and Propulsion Performance," Proceedings of the Twenty-fifth International Ocean and Polar Engineering Conference, Kona, Big Island, Hawaii, USA, June 21-26, 2015.

Lee, S. and Kim B., "A numerical study on manoeuvrability of wind turbine installation vessel using OpenFOAM,” International Journal of Naval Architecture and Ocean Engineering. Volume 7, Issue 3, May 2015.

Luquet, R., Ferrant, P., Alessandrini, B., Ducrozet, G., Gentaz, L., "Simulation of a TLP in Waves using the SWENSE scheme," Proc of ISOPE conference, 2007.

Sakamoto, N., Carrica, P.M. and Stern, F., "URANS simulations of static and dynamic manoeuvring for surface combatant: part 1. Verification and validation for forces, moment, and hydrodynamic derivatives. Journal of Marine Science and Technology, 17(4), pp.422-445, 2012.

Teng, Y., Tah, J.H., Kim, J.W., Baquet, A. Jang, H., "Tension Leg Platform Design with Consideration of Tendon Springing and Ringing," OTC-26651-MS, Kuala Lumpur, Malaysia, 2016.

Toxopeus, S.L., 2011. "Practical application of viscous-flow calculations for the simulation of manoeuvring ships," PhD. thesis, TU Delft, Delft University of Technology.

Vukcevic, V. and Jasak, H., "Embedded Free Surface Method in Polyhedral Finite Volume Framework, Part I: Computational Method," Submitted to Ocean Engineering, June 2015.

Webster, W.C., Lambrakos, K., Kim, J.W. and Jing, X., "Rod Dynamics with Large Stretch," OMAE2012-83889, Rio de Janeiro, Brazil, June 2012.

Wu, G., Jang, H., Kim, J.W., Ma, W., Wu, M.C. \& O’Sullivan, J. “Benchmark of CFD Modeling of TLP Free Motion in Extreme Event," OMAE2014-24658, San Francisco, USA, June 2014.

Wu, G., Kim, J.W., Jang, H., Baquet A., "CFD-Based Numerical Wave Basin for Global Performance Analysis," OMAE201654485, Busan, South Korea, June 2016. 